\title{
Universal width distributions in non-Markovian Gaussian processes
}

\author{
Raoul Santachiara* \\ CNRS-Laboratoire de Physique Théorique, Université Louis Pasteur, \\ 3 rue de l'Université, 67084 Strasbourg Cedex, France and \\ CNRS-Laboratoire de Physique Théorique, Ecole Normale Supérieure \\ 24 rue Lhomond, 75231 Paris Cedex 05, France \\ Alberto Rosso ${ }^{\dagger}$ \\ CNRS-Laboratoire de Physique Théorique et Modèles Statistiques \\ Bât. 100 Université Paris-Sud; 91405 Orsay Cedex, France \\ Werner Krauth ${ }^{\ddagger}$ \\ CNRS-Laboratoire de Physique Statistique \\ Ecole Normale Supérieure; 24 rue Lhomond, \\ 75231 Paris Cedex 05, France
}

(Dated: 13 Nov 2006)

\begin{abstract}
We study the influence of boundary conditions on self-affine random functions $u(t)$ in the interval $t / L \in[0,1]$, with independent Gaussian Fourier modes of variance $\sim 1 / q^{\alpha}$. We consider the probability distribution of the mean square width of $u(t)$ taken over the whole interval or in a window $t / L \in[x, x+\delta]$. Its characteristic function can be expressed in terms of the spectrum of an infinite matrix. This distribution strongly depends on the boundary conditions of $u(t)$ for finite $\delta$, but we show that it is universal (independent of boundary conditions) in the small-window limit $(\delta \rightarrow 0$, $\delta \ll \min [x, 1-x])$. We compute it directly for all values of $\alpha$, using, for $\alpha<3$, an asymptotic expansion formula that we derive. For $\alpha>3$, the limiting width distribution is independent of $\alpha$. It corresponds to an infinite matrix with a single non-zero eigenvalue. We give the exact expression for the width distribution in this case. Our analysis facilitates the estimation of the roughness exponent from experimental data, in cases where the standard extrapolation method cannot be used.
\end{abstract}

\section{INTRODUCTION}

In nature, random processes model interfaces and surfaces [1] [2], turbulent flows [3], erratic time series[4] and many other systems. In the most simple setting, these random processes correspond to a function of a scalar variable $u(t)$, and are characterized by a probability distribution $\mathcal{P}[u(t)]$ that stems from an equilibrium problem or a non-equilibrium process. The probability distribution $\mathcal{P}[u(t)]$ is often unknown, and generally inaccessible to exact analysis. In many cases, approximate methods must be brought to bear on these problems.

One of the most successful approaches in the field of random processes is the Gaussian approximation. It consists, schematically, in developing $u(t)$ on a general Fourier basis,

$$
u(t) \sim \sum_{n=-\infty}^{\infty} a_{n} \exp \left(\mathrm{i} q_{n} t\right)
$$

and in assuming that the coefficients $a_{n}=a_{-n}^{*}$ are independent Gaussian random variables. The probability distribution $\mathcal{P}[u(t)]=\mathcal{P}\left[\left\{a_{1}, a_{2}, \ldots\right\}\right]$ then factorizes into a product $\mathcal{P} \simeq \mathcal{P}^{\text {Gauss }}=\mathcal{P}\left(a_{1}\right) \mathcal{P}\left(a_{2}\right) \ldots$, where $\mathcal{P}\left(a_{n}\right)$ is a Gaussian with zero mean and variance $\sigma_{n}^{2}$. The idea behind this representation is to approximate the translation-invariant action of a complicated interacting problem by a quadratic (Gaussian) action which yields the same two-point correlation functions as in the original problem. In the particularly important case of self-affine (critical) systems, the only length scale present is the system size. The scaling of the variances with the modes $q_{n}$ can then be described by a single parameter $\alpha$ with $\sigma_{n}^{2} \propto 1 / n^{\alpha}$. In many non-trivial problems, the Gaussian approximation is in outstanding quantitative agreement with the full theory $[5,6]$, and reproduces very well even the higher-order correlation functions.

\footnotetext{
*Electronic address: raoul.santachiara@lpt.ens.fr

${ }^{\dagger}$ Electronic address: rosso@lptms.u-psud.fr

${ }_{\ddagger}$ Electronic address: krauth@lps.ens.fr
} 

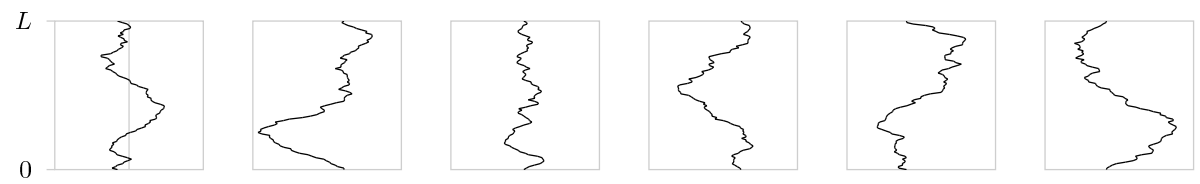

FIG. 1: Gaussian functions $u(t)$ in the interval $t / L \in[0,1]$, corresponding to a probability distribution $\mathcal{P}^{\text {Gauss }}[u(t)]$ with $\zeta=0.75(\alpha=2.5)$ (full periodic series).

Because of its decoupled Fourier modes, the Gaussian approximation is considerably simpler than the full theory and the function $u(t)$ can easily be generated through eqn (1) from Gaussian random numbers $\left\{a_{n}\right\}$ (see Fig. 1). However, the price to pay for this simplicity in Fourier space is to have non-trivial long-range correlations in real space. As we will discuss in detail, the real-space action contains generalized derivatives which, in the sense of the Riemann-Liouville derivative, can be expressed as an integral convolution with a long-range kernel [7]. For noninteger values of $\alpha / 2$, the real-space Gaussian action is non-local and the geometrical properties of the function $u(t)$ are intricate.

For $\alpha=2$, the Gaussian approximation corresponds to the notorious random walk. In this case the real-space action is local. It defines a Markovian evolution (the value of $u$ at $t+\mathrm{d} t$ depends only on the one at $t$ ). For this reason it is possible to determine in detail its geometric properties (for a recent review see [8]). For $\alpha \neq 2$, instead, the process $u(t)$ is non-Markovian: In the case $\alpha=4$ (the driven curvature model [1,9]), the evolution of the derivative $\dot{u}(t)$ is Markovian, but not the one of $u(t)$ itself. For not-integer $\alpha / 2$, the non-Markovian properties reflect the non-local character of the action. This implies that, generally, memory effects influence the shape of $u(t)$ and calculations, even within the Gaussian approximation, are difficult. In particular, the persistence exponents and the distribution of the extreme remain unknown $[10,11]$. On the other hand, the boundary conditions influence the statistical properties of $u(t)$ in a non-trivial way for all $t \in[0, L]$. Understanding these effects is important because the periodic boundary conditions for $u(t)$, which are commonplace in theoretical calculations and in numerical simulations, are usually not realized in experiments $[12,13]$.

As an example of a fundamental geometrical quantity sensitive to the boundary, we consider the mean square width

$$
w_{2}=\frac{1}{L} \int_{0}^{L} \mathrm{~d} t u^{2}(t L)-\frac{1}{L^{2}}\left[\int_{0}^{L} \mathrm{~d} t u(t L)\right]^{2},
$$

which is relevant both from the theoretical and the experimental points of view. For a self-affine random process, the mean square width is itself described by a non-trivial probability distribution $\mathcal{P}\left(w_{2}\right)$, which is neither a delta-function nor a Gaussian.

In this work, we study the influence of boundary conditions on the width distribution of $\mathcal{P}^{\text {Gauss }}[u(t)]$ for self-affine functions characterized by variances scaling with the single parameter $\alpha$. In fact, the schematic Fourier representation of eqn (1) can be rendered explicit in a number of ways in order to accommodate the boundary conditions. First it can be expanded into a full periodic series,

$$
u(t)=\sum_{n=1}^{\infty} a_{n} \cos \left(\frac{2 \pi n}{L} t\right)+b_{n} \sin \left(\frac{2 \pi n}{L} t\right) \text { (full periodic series) }
$$

where $a_{n}$ and $b_{n}$ are independent Gaussian random numbers of variance $1 / n^{\alpha}$. This implies that the function, which has zero average value, and all the derivatives are periodic, if they exist $\left(u^{(k)}(t=0)=u^{(k)}(t=L)\right.$ for $k=0,1, \cdots \leq \alpha / 2$,$) . It is also possible to expand the Gaussian function u(t)$ into a sine Fourier series,

$$
u(t)=\sum_{n=1}^{\infty} s_{n} \sin \left(\frac{\pi n}{L} t\right) \quad(\text { sine series }),
$$

again supposing that the $s_{n}$ are independent Gaussians with variance $\propto 1 / n^{\alpha}$. In that case, the function $u(t)$ vanishes at $t=0$ and $t=L$. By a uniform shift of the function, it can be made to have zero average value, as for the full periodic series. However, all the existing even derivatives vanish $\left(u^{(k)}(0)=u^{(k)}(L)=0\right.$ for $\left.k=0,2, \ldots \leq \alpha / 2\right)$ for the sine Fourier series. The full periodic series and the sine series are equivalent for the random walk $(\alpha=2)[14,15]$, but they differ for all other values of $\alpha$.

Finally, the function $u(t)$ can also be expanded into a cosine Fourier series,

$$
u(t)=\sum_{n=1}^{\infty} c_{n} \cos \left(\frac{\pi n}{L} t\right) \quad(\text { cosine series }),
$$


again with $\sigma^{2}\left(c_{n}\right) \propto 1 / n^{\alpha}$. In this case, the random function is not forced to satisfy $u(0)=u(L)$, and for this reason it has been used to study free random walks [8]. Analogously to the sine Fourier series, all the odd derivatives of $u(t)$ must vanish at the boundaries, if they exist $\left(u^{(k)}(0)=u^{(k)}(L)=0\right.$ for $\left.k=1,3, \ldots \leq \alpha / 2\right)$. This paper shows that these additional constraints strongly influence the geometry of the function $u(t)$ for $\alpha \neq 2$.

The above three series representations correspond to the same real-space action $\mathcal{S}[u(t)]$ and its associated Gaussian probability distribution $\mathcal{P}^{\text {Gauss }}[u(t)]$

$$
\mathcal{S}[u(t)]=\frac{1}{2} \int_{0}^{L} \mathrm{~d} t\left(\frac{\partial^{\alpha / 2} u(t)}{\partial t^{\alpha / 2}}\right)^{2} \Longrightarrow \mathcal{P}^{\text {Gauss }}[u(t)]=\exp \{-\mathcal{S}[u(t)]\} .
$$

For non-integer values of $\alpha / 2$, the generalized derivative in eqn (5) is defined in momentum space: $\left[\partial^{\alpha / 2} / \partial t^{\alpha / 2}\right] \mathrm{e}^{\mathrm{i} q t}=$ $(\mathrm{i} q)^{\alpha / 2} e^{\mathrm{i} q t}$ (the derivative can also be defined in real space [7]). Using this definition we can compute the variances of the Fourier coefficients:

$$
\left[\sigma_{n}^{\text {per }}\right]^{2}=\frac{L^{\alpha-1}}{2^{\alpha-1} \pi^{\alpha} n^{\alpha}} \Longrightarrow \mathcal{P}^{\text {Gauss }}\left[\left\{a_{n}, b_{n}\right\}\right]=\prod_{n=1}^{\infty} \frac{1}{2 \pi \sigma_{n}^{2}} \exp \left[-\frac{1}{2} \frac{\left(a_{n}^{2}+b_{n}^{2}\right)}{\sigma_{n}^{2}}\right] \quad \text { (full periodic series). }
$$

For the sine and the cosine series, the variances are

$$
\left[\sigma_{n}^{\cos }\right]^{2}=\left[\sigma_{n}^{\sin }\right]^{2}=\frac{2 L^{\alpha-1}}{\pi^{\alpha} n^{\alpha}}
$$

This choice leads to analogous expressions for the probability distributions $\mathcal{P}^{\text {Gauss }}\left[\left\{s_{n}\right\}\right]$ and $\mathcal{P}^{\text {Gauss }}\left[\left\{c_{n}\right\}\right]$, respectively.

The variances in eqns (6) and (7) scale with the system size as $\propto L^{\alpha-1}=L^{2 \zeta}$ where $\zeta$ is the roughness exponent. This exponent characterizes the main geometric properties of a self-affine system.

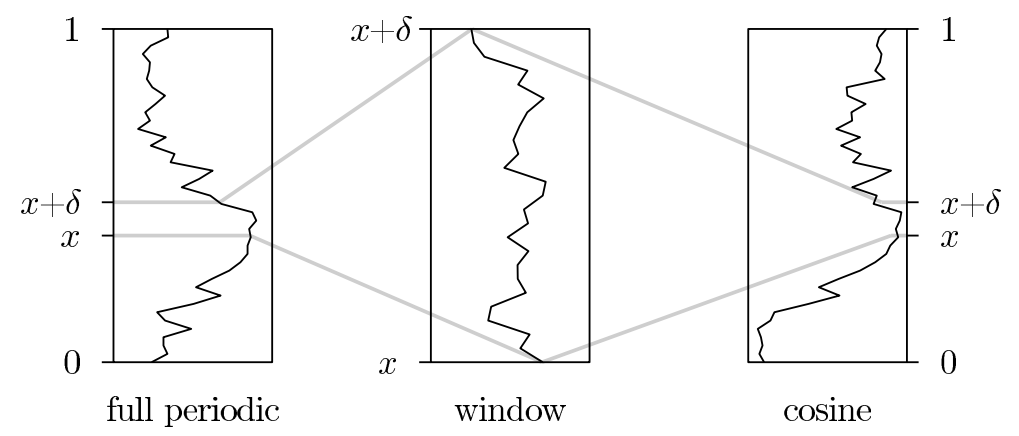

FIG. 2: Gaussian functions $u(t / L)$ corresponding to the full periodic and the cosine series. Window boundary conditions correspond to picking out a piece of $u(t)$ in the interval $t / L \in[x, x+\delta]$ and shifting $u(t / L)$ such that the window mean value vanishes.

In this paper, we first consider Gaussian functions on the entire interval $[0, L]$. We compute the average of the width distribution for the three series and for general values of $\alpha$ (Section II A). The average of the width distribution strongly depends on the boundary conditions (for $\alpha \neq 2$ ). These results are then generalized (Section II B) to the case when the function $u(t)$ is restricted to a window of width $\delta$, in the interval $t / L \in[x, x+\delta]$ :

$$
w_{2}(x, \delta)=\frac{1}{\delta} \int_{x}^{x+\delta} \mathrm{d} t u^{2}(t L)-\left(\frac{1}{\delta} \int_{x}^{x+\delta} \mathrm{d} t u(t L)\right)^{2} .
$$

These window boundary conditions are closer to the experimental situation than those realized by either the full periodic or the cosine series (see Fig. 2). We write (Section III) the characteristic function of the width distribution in terms of the eigenvalues of an infinite matrix which depends on the basis functions of the Fourier series, and on the window parameters $\{\delta, x\}$. Practically, to compute the width distribution for any of the series, in an arbitrary finite window, it suffices to compute this characteristic function from the eigenvalues of a finite-rank approximation of the above matrix, and to perform an inverse Fourier transform. It is easy to see that the width distribution depends on the size of the window and on the choice of boundary conditions. In the small-window limit $(\delta \rightarrow 0$ and far from the boundaries $\delta \ll \min [x, 1-x]$ ), finite-rank approximations to the above matrix cease to be accurate (the limit of 

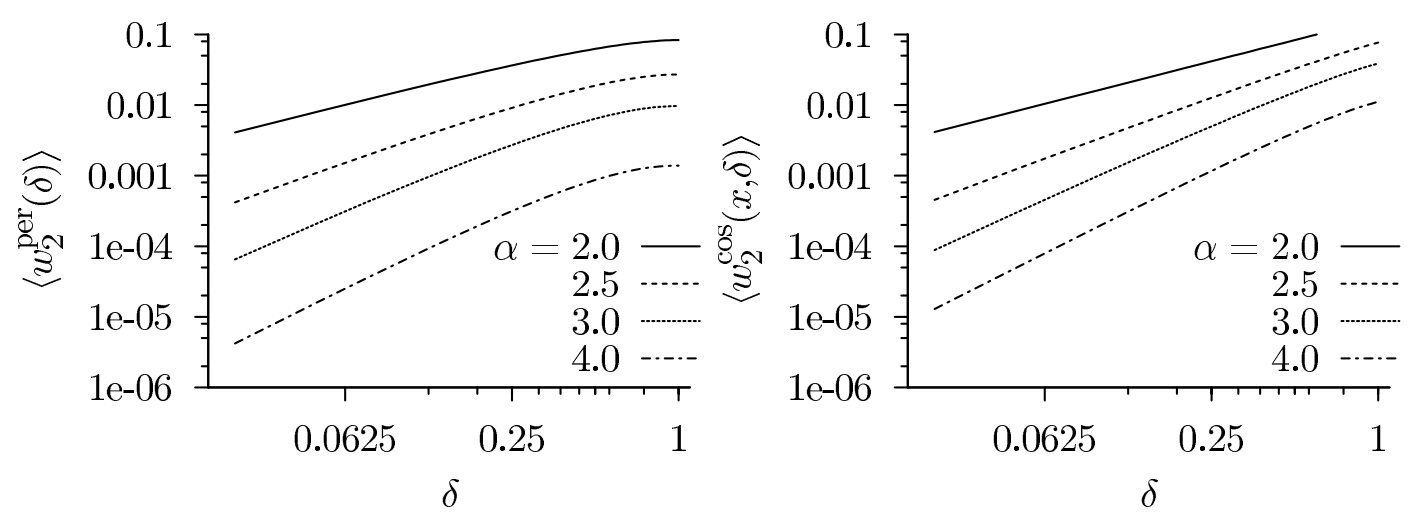

FIG. 3: Mean square width as a function of window width, for different values of $\alpha$, for the full periodic series (left) and the cosine series (right, with $x=\frac{1}{2}(1-\delta)$ ) (from eqns (11) and (16)).

rank $N \rightarrow \infty$ does not commute with the limit $\delta \rightarrow 0$, for $\alpha<3$ ), but we are able to write all the cumulants of the width distribution distribution directly in this limit (Section III C) via a subtle asymptotic expansion in powers of $\delta$. We prove that in the small-window limit the width distribution becomes independent of the boundary condition. We also show how to practically compute the ( $\alpha$-dependent) universal width distribution directly in this limit. For $\alpha>3$ (Section III C 2), the problem simplifies. The characteristic function in the small-window limit then corresponds to a matrix with only one non-zero eigenvalue, and the corresponding width distribution no longer depends on $\alpha$. We give its explicit form. Finally, we compute the logarithmic corrections to these asymptotic results for odd-integer values of $\alpha$. In Appendix A and Appendix B, we provide technical details of our calculation.

In a previous paper on the same subject [16], we already studied the second moment of the width distribution, and presented arguments for the universality in the small-window limit. The more complete, and more concrete, calculations of the present paper rely on the representation of the characteristic function in terms of the spectrum of a matrix, which was not contained in [16].

\section{AVERAGE WIDTH}

In a self-affine (critical) system, the length $L$ of the total interval is, as mentioned, the only characteristic length and the average value of the total width scales as $\left\langle w_{2}\right\rangle \propto L^{\zeta}(\langle\ldots\rangle$ denotes the ensemble average).

\section{A. Average width for $\delta=1$}

Fixing $\delta=1$ and $x=0$ in eqn (8) and integrating over $t$ yields

$$
w_{2}= \begin{cases}\frac{1}{2} \sum_{n=1}^{\infty}\left(a_{n}^{2}+b_{n}^{2}\right) & \text { (full periodic series) } \\ \sum_{n=1}^{\infty}\left[\frac{1}{2} s_{n}^{2}-\sum_{m=1}^{\infty} \frac{\left(1-(-1)^{m}\right)\left(1-(-1)^{n}\right) s_{n} s_{m}}{\pi^{2} n m}\right] & \text { (sine series) } \\ \frac{1}{2} \sum_{n=1}^{\infty} c_{n}^{2} & \text { (cosine series). }\end{cases}
$$

From the Gaussian probability distributions given in eqn (5)-eqn (7), the ensemble averages are

$$
\frac{\left\langle w_{2}\right\rangle}{L^{\alpha-1}}= \begin{cases}\frac{2}{(2 \pi)^{\alpha}} \zeta(\alpha) & \text { (full periodic series) } \\ \frac{1}{\pi^{\alpha}} \zeta(\alpha)-\frac{2}{\pi^{2}} \frac{2^{\alpha+2}-1}{(2 \pi)^{\alpha}} \zeta(\alpha+2) & \text { (sine series) } \\ \frac{1}{\pi^{\alpha}} \zeta(\alpha) & \text { (cosine series) }\end{cases}
$$

where $\zeta(x)=\sum_{n=1}^{\infty} 1 / n^{x}$ is the Riemann Zeta function. For all $\alpha$, the average width of the full periodic series is smaller than that of the cosine series, as is quite natural. For $\alpha=2$, the average widths of the full periodic series and the sine series coincide, because the two differ only through boundary conditions for the derivative of $u$, that a Markovian process is insensitive to. 


\section{B. Average width for $\delta<1$}

We now compute the average width for $\delta<1$ for the full periodic series, the cosine, and the sine series. The width $w_{2}^{\text {per }}(\delta)$ is independent of the origin $x$ but the $x$-dependence of the average width cannot be neglected in the other two cases. For the full periodic series, one obtains from eqns (2) and (8)

$$
w_{2}^{\mathrm{per}}(\delta)=\sum_{n, m=1}^{\infty} a_{n} a_{m} C_{n m}(\delta)+a_{n} b_{m} I_{n m}(\delta)+b_{n} b_{m} S_{n m}(\delta)
$$

where the coefficients $C_{n m}(\delta)$ are given by elementary integrals:

$$
C_{m n}=\frac{1}{\delta} \int_{x}^{x+\delta} \mathrm{d} t \cos (2 \pi m t) \cos (2 \pi n t)-\frac{1}{\delta^{2}} \int_{x}^{x+\delta} \mathrm{d} t \cos (2 \pi m t) \int_{x}^{x+\delta} \mathrm{d} t \cos (2 \pi n t) .
$$

Analogously, $S_{n m}$ and $I_{n m}$ can be expressed in terms of sine-sine and cosine-sine integrals. For the full periodic series, these coefficients are naturally independent of $x$.

Equation (9) allows to compute $w_{2}^{\text {per }}$ for one given sample. Integrating over the Gaussian Fourier components $\left\{a_{n}, b_{n}\right\}$, we get

$$
\left\langle w_{2}^{\text {per }}(\delta)\right\rangle=\frac{L^{\alpha-1}}{2^{\alpha-1} \pi^{\alpha}} \sum_{n=1}^{\infty} \frac{C_{n n}+S_{n n}}{n^{\alpha}}, \text { where } C_{n n}+S_{n n}=1-\frac{1-\cos (2 \pi n \delta)}{2(\pi n \delta)^{2}} .
$$

The sum in eqn (11) is easily evaluated for finite $\delta$ (see Fig. 3). The limit of this mean value for $\delta \rightarrow 0$ cannot be obtained by a naive Taylor expansions of each term in this infinite sum in eqn (11), because it is not uniformally convergent in the interval $\delta \in[0,1]$ for $\alpha<3$ (in the limit $\delta \rightarrow 0$, the terms of the sum behave as $\left(C_{n n}(\delta)+\right.$ $\left.S_{n n}(\delta)\right) n^{-\alpha} \sim \delta^{2} n^{2-\alpha}$, producing a diverging series for $\alpha<3$ ). As we will discuss in detail later, the higher cumulants of the width distribution are given by multiple infinite sums which present the same pathology as the sum in eqn (11). To sum the series in the limit $\delta \rightarrow 0$, we have derived a very useful expansion formula which has the same structure as the Euler-Maclaurin formula:

$$
\sum_{n=1}^{\infty} \frac{f(n \delta)}{n^{\alpha}}=\delta^{\alpha-1} \int_{0}^{\infty} \mathrm{d} t\left(\frac{f(t)}{t^{\alpha}}-\sum_{m=0}^{\lfloor\alpha\rfloor-1} \frac{f^{(m)}(0) t^{m-\alpha}}{m !}\right)+\sum_{m=0}^{\infty} \delta^{m} f^{(m)}(0) \frac{\zeta(\alpha-m)}{m !}
$$

where $\lfloor\alpha\rfloor$ is the integer part of $\alpha$. Equation (12) holds inside the convergence radius $\delta=1$ for all the quantities considered in this work (the formula is proved in Appendix A). For analytic functions $f(z)$ and non-integer $\alpha$, the first term on the right can be interpreted as the naive limit of the sum as $\delta \rightarrow 0$, with $t=n \delta$, whereas the second term contains the Taylor expansion of $f(n \delta)$ around zero. For integer $\alpha$, the singularity of $\zeta(1)$ generates additional logarithms (see Appendix A, again). Using the expansion formula of eqn (12), one arrives at:

$$
\frac{\left\langle w_{2}^{\mathrm{per}}(\delta)\right\rangle}{L^{\alpha-1}}=\frac{2^{-\alpha-1}}{\zeta(-\alpha-1)} \frac{\zeta(\alpha+2)}{\pi^{\alpha+2}} \delta^{\alpha-1}+\frac{4}{(2 \pi)^{\alpha}} \sum_{n=1}^{\infty}(-1)^{n+1} \frac{\zeta(\alpha-2 n)}{(2 n+2) !}(2 \pi \delta)^{2 n} .
$$

To take into account the logarithmic corrections for odd integer $\alpha$ we must use eqn (A6) instead of eqn (12). The final result writes:

$$
\frac{\left\langle w_{2}^{\text {per }}(\delta)\right\rangle}{L^{\alpha-1}}=(-1)^{\frac{\alpha+1}{2}} \frac{\psi_{0}(\alpha+2)-\log (2 \pi \delta)}{2 \pi(\alpha+1) !} \delta^{\alpha-1}+\frac{4}{(2 \pi)^{\alpha}} \sum_{n \neq \frac{\alpha-1}{2}}(-1)^{n+1} \frac{\zeta(\alpha-2 n)}{(2 n+2) !}(2 \pi \delta)^{2 n} \quad(\alpha \text { odd integer })
$$

where $\psi_{0}(z)$ is the digamma function. An expansion analogous to eqn (13) appears in the correlation function governing the density of zero-crossings of a Gaussian function [10]. For integer even $\alpha$, the series in eqn (13) is finite (because $\zeta(x)=0$ for $x=\{-2,-4, \ldots\})$. The mean square widths for the periodic random walk $(\alpha=2)$ and the driven curvature model $(\alpha=4)$ then have compact expressions:

$$
\frac{\left\langle w_{2}^{\text {per }}(\delta)\right\rangle}{L^{\alpha-1}}= \begin{cases}\frac{1}{6} \delta-\frac{1}{12} \delta^{2} & \text { for } \alpha=2 \\ \frac{1}{144} \delta^{2}-\frac{1}{120} \delta^{3}+\frac{1}{360} \delta^{4} & \text { for } \alpha=4\end{cases}
$$


For the cosine series, we obtain, from eqns (4) and (8), the mean squared width

$$
w_{2}^{\cos }(x, \delta)=\sum_{n, m=1}^{\infty} c_{n} c_{m} D_{n m}(x, \delta),
$$

where the coefficients are given by a symmetric matrix

$$
D_{m n}(x, \delta)=\frac{1}{\delta} \int_{x}^{x+\delta} \mathrm{d} t \cos (\pi m t) \cos (\pi n t)-\frac{1}{\delta^{2}} \int_{x}^{x+\delta} \mathrm{d} t \cos (\pi m t) \int_{x}^{x+\delta} \mathrm{d} t \cos (\pi n t) .
$$

$D_{n m}$ is the overlap matrix of the basis functions in eqn (4) on the interval $[x, x+\delta]$. For $\delta=1$, the basis functions are, by construction, an orthonormal set, and $D_{n m}=\frac{1}{2} \delta_{n m}$.

Integrating over the Gaussian variable $\left\{c_{n}\right\}$, the average width $\left\langle w_{2}^{\text {cos }}\right\rangle$ becomes

$$
\left\langle w_{2}^{\cos }(x, \delta)\right\rangle=\frac{2 L^{\alpha-1}}{\pi^{\alpha}} \sum_{n=1}^{\infty} \frac{D_{n n}(x, \delta)}{n^{\alpha}} .
$$

The sum in eqn (16) is again easily evaluated, for any value of $x$ and $\delta$. The behavior of $\left\langle w_{2}^{\cos }(x, \delta)\right\rangle$ as a function of $\delta$ is shown in Fig. 3 for $x=\frac{1}{2}(1-\delta)$. For small $\delta$ we notice that $\left\langle w_{2}^{\cos }(x, \delta)\right\rangle \propto \delta^{\alpha-1}$ for $\alpha<3$ and $\left\langle w_{2}^{\cos }(x, \delta)\right\rangle \propto \delta^{2}$ for $\alpha>3$. A special case is again $\alpha=2$ where we have $\left\langle w_{2}^{\cos }(x, \delta)\right\rangle \propto \delta$ for all $\delta$.

\section{Small-window limit}

In the following, we extract the universal behavior of $\left\langle w_{2}^{\cos }(x, \delta)\right\rangle$ for a small window $[x, x+\delta]$ far from the boundaries (for $\delta \ll \min (x, 1-x)$ ). For $\alpha<3$, eqn (16) can be expanded as

$$
\left\langle w_{2}^{\cos }(x, \delta)\right\rangle=\frac{2 L^{\alpha-1}}{\pi} \delta^{\alpha-1} \int_{0}^{\infty} \mathrm{d} t \frac{D(t, x / \delta)}{t^{\alpha}}+O\left(\delta^{2}\right),
$$

where the function $D(t, x / \delta)$ is defined through $D(\pi n \delta, x)=D_{n n}(x)$. We write $D(t, x / \delta)$ as a sum of two terms:

$$
D(t, x / \delta)=h(t, x / \delta)+\frac{1}{2}[C(t)+S(t)] .
$$

The function $h(t, x / \delta)$ contains all the $x$-dependence of $D(t, x / \delta)$ :

$$
h\left(t, \frac{x}{\delta}\right)=\cos \left(2 t\left(\frac{x}{\delta}+\frac{1}{2}\right)\right)\left[\frac{\sin (2 t)}{4 t}-\frac{1}{t^{2}} \sin \left(\frac{t}{2}\right)^{2}\right] .
$$

For $x / \delta \gg 1, h(t, x / \delta)$ oscillates rapidly and gives a vanishing contribution to the integral in eqn (17). In particular, we can show that

$$
\lim _{x / \delta \rightarrow \infty} \frac{\left\langle w_{2}^{\cos }(x, \delta)\right\rangle}{L^{\alpha-1}}=\frac{2^{-\alpha-1}}{\zeta(-\alpha-1)} \frac{\zeta(\alpha+2)}{\pi^{\alpha+2}} \delta^{\alpha-1}+O[\exp (-x / \delta)] .
$$

Comparing the above expression with eqn (13), we conclude that, for $\alpha<3$, the dominant contributions to $\left\langle w_{2}^{\cos }(x, \delta)\right\rangle$ and to $\left\langle w_{2}^{\text {per }}(\delta)\right\rangle$ coincide for small $\delta$ (compare also with Fig. 3). This conclusion can be extended to the sine series, where the explicit $x$-dependence is again due to oscillatory terms that vanish in the small-window limit.

As mentioned before, the series in eqn (16) is uniformly convergent for $\alpha>3$, so that one can take the limit $\delta \rightarrow 0$ for each mode and then do the sum. Using the expansion

$$
D_{n m}(x)=\frac{1}{12} n m \pi^{2} \sin (\pi m x) \sin (\pi n x) \delta^{2}+O\left(\delta^{4}\right)
$$

in eqn (16), we obtain

$$
\frac{\left\langle w_{2}^{\cos }(x, \delta)\right\rangle}{L^{\alpha-1}}=\frac{1}{6}\left[\sum_{n=1}^{\infty} \frac{\sin ^{2}(n \pi x)}{n^{\alpha-2}}\right] \pi^{2-\alpha} \delta^{2}+O\left(\delta^{\alpha-1}, \delta^{4}\right) .
$$

For $x=0$ and $x=1$ the $\delta^{2}$ term vanishes, while for $0<x<1$ it is smaller than the corresponding term for the periodic case. This behavior is consistent with the fact that, for $\alpha>2$, the cosine series imposes vanishing derivatives at the end points. Hence these boundary conditions force $\left\langle w_{2}^{\text {cos }}\right\rangle$ to be smaller than $\left\langle w_{2}^{\text {per }}\right\rangle$ for all $0<x<1$. The sine series gives a result analogous to the one for the cosine series. It suffices to replace the sine in the sum of eqn (19) by a cosine. 


\section{WIDTH DISTRIBUTION}

The scaling behavior of the average width gives access to the value of $\alpha$ and thus to the roughness exponent $\zeta=(\alpha-1) / 2$. However, the value of this exponent only depends on the 2-point correlation functions, and captures no finer geometric properties of the function $u(t)$. To discriminate between a Gaussian and a non-Gaussian function, one must have access to higher cumulants, as they are contained in the sample-to-sample fluctuations of the two-point correlation functions. The distribution $\mathcal{P}\left(w_{2}\right)$ has been used to analyze numerical and experimental data [13, 2023 . The width distribution also allows to estimate the roughness exponent from experimental data which are not sufficiently good to plot $\left\langle w_{2}(\delta)\right\rangle$ vs $\delta$ over several orders of magnitude in $\delta$.

For general values of $\alpha$, the width distribution has been computed for the entire interval $(\delta=1, x=0)$, for the full periodic series, [17-19], and for the cosine series [13]. The underlying simplification with respect to the calculations in the present paper is that the matrix of coefficients for $\delta=1$ satisfies $I_{n m}=0$ while $C_{n m}, S_{n m}$ and $D_{n m}$ are diagonal, as evident in eqns (10) and (15). In order to study window boundary conditions (but also for the sine series), the previous framework must be generalized to non-diagonal matrices.

The width distribution can be obtained from the symmetric matrices

$$
A_{n m}^{\cos }=2 \sigma_{n}^{\cos } D_{n m} \sigma_{m}^{\cos } \text {. }
$$

and

$$
A^{\text {per }}=\left(\begin{array}{cc}
2 \sigma_{n}^{\text {per }} C_{n m} \sigma_{m}^{\text {per }} & \sigma_{n}^{\text {per }} I_{n m} \sigma_{m}^{\text {per }} \\
\sigma_{n}^{\text {per }} I_{m n} \sigma_{m}^{\text {per }} & 2 \sigma_{n}^{\text {per }} S_{n m} \sigma_{m}^{\text {per }}
\end{array}\right)
$$

respectively. Concretely (see eqns (9) and (14)), individual realizations of the mean square width distribution are generated by multiplying the matrices $A$ in eqns (20) and (21) by vectors of normal distributed Gaussian variables. This allows to obtain $\mathcal{P}\left(z=w_{2} /\left\langle w_{2}\right\rangle\right)$ approximately through direct simulation (see [15]).

On the other hand, one can compute all the moments of the distribution from contractions of a given matrix $A$ (which can stand for $A^{\text {cos }}, A^{\text {sin }}$, or $A^{\text {per }}$ ). For example, the second moment of the width distribution is given by

$$
\mu_{2}=\left\langle w_{2}^{2}\right\rangle=\frac{1}{2} \sum_{n, m} A_{n m}^{\cos } A_{m n}^{\cos }+\frac{1}{4}\left(\sum_{n} A_{n n}^{\cos }\right)^{2}=\frac{1}{2} \operatorname{Tr}\left[A^{\cos }\right]^{2}+\left[\frac{1}{2} \operatorname{Tr} A^{\cos }\right]^{2} .
$$

Similar expressions exist for higher moments of the width distribution. As shown in Appendix B, the cumulant $\kappa_{l}$ of the rescaled width distribution can be expressed in a simpler way than the moments, as a trace of the matrix $A$ taken to the $l$ th power:

$$
\kappa_{l}=\frac{(l-1) !}{2\left\langle w_{2}\right\rangle^{l}} \operatorname{Tr}\left[A^{l}\right]
$$

This is already apparent in eqn (22), where $\mu_{2}=\left\langle w_{2}\right\rangle^{2}\left(\kappa_{2}+\kappa_{1}^{2}\right)$. The cumulants are thus given in terms of the eigenvalues $\left\{\lambda_{1}, \lambda_{2}, \ldots\right\}$ of the matrix $A$ as $\operatorname{Tr} A^{l}=\sum_{k} \lambda_{k}^{l}$ :

$$
\kappa_{l}=\frac{(l-1) !}{2} \frac{\sum_{k} \lambda_{k}^{l}}{\left(\sum_{k} \lambda_{k}\right)^{l}} .
$$

The cumulants in eqn (23) yield an explicit formula for the cumulant-generating function $\Psi\left(s /\left\langle w_{2}\right\rangle\right)$, where

$$
\Psi(s)=\sum_{k=1}^{\infty} \sum_{l=1}^{\infty} \frac{\lambda_{k}^{l}}{2 l} s^{l}=\sum_{k} \frac{1}{2} \log \left(1-\lambda_{k} s\right) .
$$

The characteristic function $f(s)=\exp (\Psi(\mathrm{i} s))$, the exponential of the cumulant-generating function $\Psi(\mathrm{i} s)$, is given by

$$
f(s)=\prod_{k} \frac{1}{\sqrt{1-\mathrm{i} \lambda_{k} s}} .
$$

The spectrum of the matrices under consideration is such that the infinite product in eqn (24) is uniformally convergent. The associated width distribution is recovered through an inverse Fourier transform,

$$
\mathcal{P}\left(z=w_{2} /\left\langle w_{2}\right\rangle\right)=\frac{1}{2 \pi} \int_{-\infty}^{\infty} \mathrm{d} s \exp (-\mathrm{i} z s) f(s),
$$

which can be obtained by straightforward Riemann integration because the branch points $s_{k}=\mathrm{i} / \lambda_{k}$ are away from the real axis. 

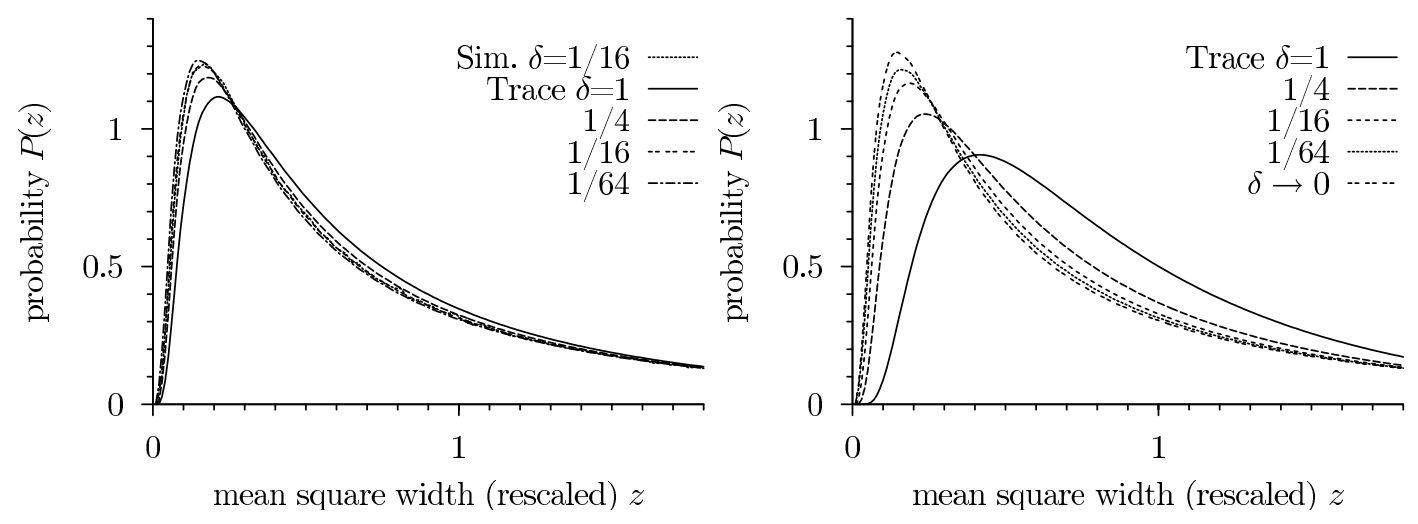

FIG. 4: Rescaled probability distributions for the mean square width for $\zeta=0.75(\alpha=2.5)$ for the cosine series (left) and the full periodic series (right), obtained from eqns (24) and (25). The result of direct simulations for $\delta=1 / 16$ (from eqn (14)), and the solution in the limit $\delta \rightarrow 0$ (from the matrix in eqn (32)) are also shown. Sizes of matrices are $N=512$ and $N=1024$.

\section{A. Width distribution for the entire interval $(\delta=1)$}

As mentioned above, in the case $\delta=1$, the matrices

$$
A^{\text {per }}=\left(\begin{array}{cc}
{\left[\sigma_{n}^{\text {per }}\right]^{2} \delta_{n m}} & 0 \\
0 & {\left[\sigma_{n}^{\text {per }}\right]^{2} \delta_{n m}}
\end{array}\right), \quad \text { and } \quad A^{\text {cos }}=\left[\sigma_{n}^{\cos }\right]^{2} \delta_{n m},
$$

are diagonal and the computation of the cumulants from eqn (23) is direct:

$$
\kappa_{l}= \begin{cases}(l-1) ! \zeta(l \alpha) / \zeta(\alpha)^{l} & \text { (full periodic series) } \\ (2 l-2) ! ! \zeta(l \alpha) / \zeta(\alpha)^{l} & \text { (cosine series) }\end{cases}
$$

The cumulants for the sine series are more complicated because the corresponding matrix $A^{\text {sin }}$ presents non-diagonal terms coming from the non-vanishing mean $\frac{1}{L} \int_{0}^{L} \mathrm{~d} t \sin (\pi n t / L)$ for odd values of $n$. The second cumulant, for example, is given by

$$
\kappa_{2}^{\text {sin }}=\frac{2 \zeta(2 \alpha)+2^{-2 \alpha+1}\left(16 \pi^{-4}\left(2^{\alpha+2}-1\right)^{2} \zeta(\alpha+2)^{2}-8 \pi^{-2}\left(2^{2 \alpha+2}-1\right) \zeta(2 \alpha+2)\right)}{\left[\zeta(\alpha)-2^{-\alpha+1} \pi^{-2}\left(2^{\alpha+2}-1\right) \zeta(\alpha+2)\right]^{2}} .
$$

$\kappa_{2}^{\text {sin }}$ agrees with $k_{2}^{\text {per }}$ only for $\alpha=2$, as expected. In the case of the full periodic and the cosine series, the characteristic functions assume a simple form

$$
f(s)= \begin{cases}\prod_{k}\left(1-\mathrm{i} L^{\alpha-1}(2 \pi)^{\alpha} k^{-\alpha} s\right)^{-1} & (\text { full periodic series }) \\ \prod_{k}\left(1-\mathrm{i} L^{\alpha-1} \pi^{\alpha} k^{-\alpha} s\right)^{-\frac{1}{2}} & \text { (cosine series) }\end{cases}
$$

As already discussed in [19], the two-fold degeneracy of the spectrum of $A^{\text {per }}$ for $\delta=1$, evident in eqn (26), yields a characteristic function $f(s)$ with simple poles on the imaginary axis. This simplifies the inverse Fourier transformation.

\section{B. Width distribution for windows (finite $\delta$ )}

The expressions allowing to recover the width distribution from the eigenvalues of the matrices $A^{\text {cos }}, A^{\text {per }}$, and $A^{\text {sin }}$ remains valid for intervals $\delta<1$, even though the computer must now be used for finite approximations of these infinite matrices, which are no longer diagonal. These calculations can be easily checked by direct simulation, as mentioned before. The outcome of this analysis is shown in Fig. 4 for the case $\alpha=5 / 2$. It is evident that the width distribution changes with the sample size for the value of $\alpha$ chosen. Furthermore, the direct evaluation of the rescaled width distribution for finite but small $\delta$ suggests that this distribution becomes universal (independent of the boundary conditions) in the limit $\delta \rightarrow 0$. This scenario is confirmed by comparing the evolution of the (normalized) spectra of $A^{\text {per }}$ and $A^{\text {cos }}$, as shown in Fig. 5 . 


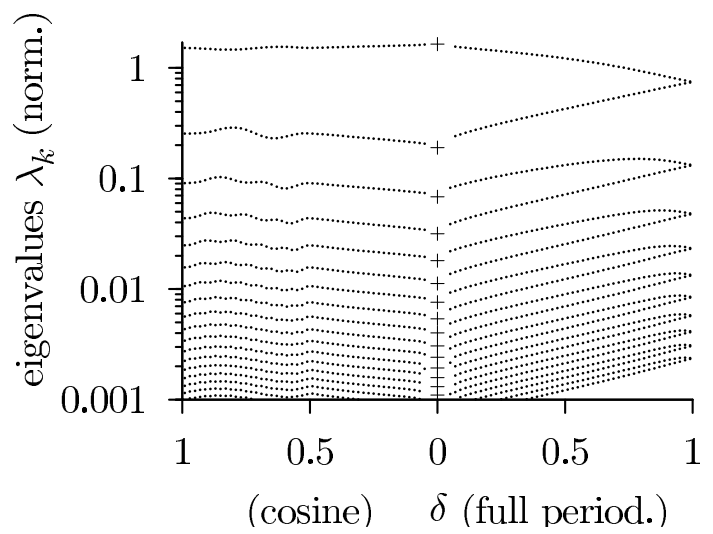

FIG. 5: Eigenvalues $\left\{\lambda_{1}, \lambda_{2}, \ldots\right\}$ for the cosine and the full periodic series as a function of window size $\delta(\alpha=2.5(\zeta=0.75)$, for the cosine series, $x=\frac{1}{2}(1-\delta)$ ). The spectrum in the small-window limit $\delta \rightarrow 0$ (crosses), is obtained in Section III C. Eigenvalues are normalized as $\sum_{k} \lambda_{k}=2$.

We then compute $\mathcal{P}\left(z=w_{2} /\left\langle w_{2}\right\rangle\right)$ directly in the limit $\delta \rightarrow 0$. However, for small $\delta$, increasingly larger matrices $A^{\text {per }}$ and $A^{\text {cos }}$ must be considered because of the non-uniform convergence of the traces of these matrices for $\delta \in[0,1]$.

In Section III C we determine directly the width distribution at the limit $\delta \rightarrow 0$. In particular, analogously to the computation of asymptotics of the average width (Section II C), the expansion formula eqn (12) is proven useful to compute the cumulants of the distribution in the small-window limit for $\alpha<3$ (Section III C 1). This analysis serves two purposes: it proves the universality of the width distribution and provides a high-precision method to compute it directly in the limit $\delta \rightarrow 0$.

\section{Width distribution in the small-window limit}

In Section II C we computed the mean square widths $\left\langle w_{2}^{\text {per }}(\delta)\right\rangle$ and $\left\langle w_{2}^{\text {cos }}(x, \delta)\right\rangle$ in the small-window limit. We now determine the rescaled width distribution $\mathcal{P}(z)$ in this limit.

\section{Small-window limit $(\alpha<3)$}

The computation of $\kappa_{l}$ as a $\delta$-expansion is along the lines of the previous determination of $\left\langle w_{2}\right\rangle$. For $\alpha<3$, we handle the non-uniform convergence of the traces in eqn (23) for $\delta \rightarrow 0$ using the expansion formula eqn (12). The leading contribution to $\kappa_{l}, l=1,2, \ldots$, is given by multiple integrals:

$$
\begin{gathered}
\kappa_{l}^{\text {per }}=\frac{\pi^{l(\alpha-1)}(l-1) !}{2 \widehat{w}_{2}^{l}} \int_{0}^{\infty} \cdots \int_{0}^{\infty} \prod_{n=1}^{l} \frac{\mathrm{d} t_{n}}{t_{n}^{\alpha}} \operatorname{Tr}\left[A^{\text {per }}\left(t_{1}, t_{2}\right) A^{\text {per }}\left(t_{2}, t_{3}\right) \cdots A^{\text {per }}\left(t_{l}, t_{1}\right)\right]+O\left(\delta^{3-\alpha}\right) \\
\kappa_{l}^{\cos }(x / \delta)=\frac{\pi^{l(\alpha-1)}(l-1) !}{2 \widehat{w}_{2}^{l}} \int_{0}^{\infty} \cdots \int_{0}^{\infty} \prod_{n=1}^{l} \frac{\mathrm{d} t_{n}}{t_{n}^{\alpha}}\left[A^{\cos }\left(t_{1}, t_{2}, \frac{x}{\delta}\right) A^{\cos }\left(t_{2}, t_{3}, \frac{x}{\delta}\right) \cdots A^{\cos }\left(t_{l}, t_{1}, \frac{x}{\delta}\right)\right]+O\left(\delta^{3-\alpha}\right) .
\end{gathered}
$$

In the above equations, $\widehat{w}_{2}$ is the prefactor of the leading term in the expansion of $\left\langle w_{2}(\delta, x)\right\rangle$, given in eqn $(13)$ :

$$
\widehat{w}_{2}=\frac{2^{-\alpha-1}}{\zeta(-\alpha-1)} \frac{\zeta(\alpha+2)}{\pi^{\alpha+2}}
$$

which is independent of the boundary conditions. $A^{\cos }\left(t_{n}, t_{n+1}, x / \delta\right)$ and $A^{\text {per }}\left(t_{n}, t_{n+1}\right)$ are obtained by replacing $\pi n \delta$ with $t_{n}$ in $A^{\text {cos }}$ and in $A^{\text {per }}$. We now verify for each cumulant

$$
\kappa_{l}^{\cos }(x / \delta \rightarrow \infty) \rightarrow \kappa_{l}^{\text {per }}+O\left(e^{-x / \delta}\right) .
$$

Extracting the $x / \delta$ independent part of $\kappa_{l}^{\cos }$ one obtains

$$
\kappa_{l}^{\cos }=\frac{\pi^{l(\alpha-1)}(l-1) !}{2 \widehat{w}_{2}^{l}} \int_{0}^{\infty} \cdots \int_{0}^{\infty} \prod_{n=1}^{l} \frac{\mathrm{d} t_{n}}{t_{n}^{\alpha}} \operatorname{Tr}\left[\tilde{A}^{\cos }\left(t_{1}, t_{2}\right) \tilde{A}^{\cos }\left(t_{2}, t_{3}\right) \cdots \tilde{A}^{\cos }\left(t_{l}, t_{1}\right)\right]+\text { oscill. terms }
$$




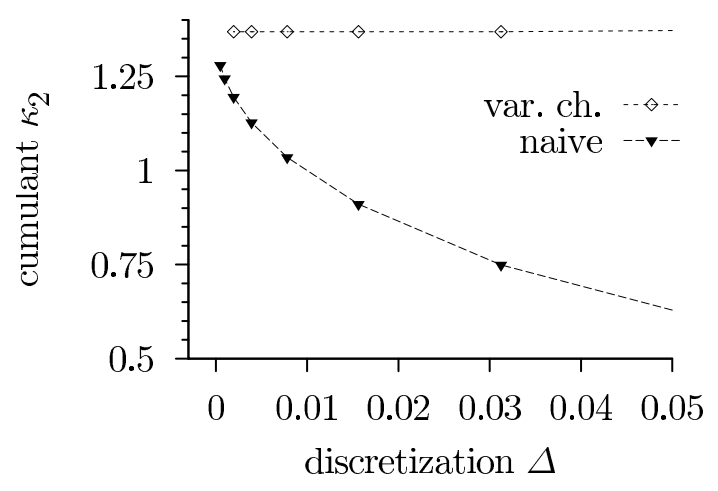

FIG. 6: Cumulant $\kappa_{2}$ of the rescaled width distribution for $\alpha=2.5$ from a naive discretization of the integral in eqn (30), and after the change of variables in eqn (32) (Riemann integration in the interval $t \in[0,15]$, using $\Delta=t_{i+1}-t_{i}$ ).

where $\tilde{A}^{\text {cos }}$ is now independent of $x$ and given by

$$
\tilde{A}^{\cos }\left(t, t^{\prime}\right)=\frac{4 L^{\alpha-1}}{\pi^{\alpha}}\left(\begin{array}{l}
\frac{1}{2\left(t-t^{\prime}\right)} \sin \frac{t-t^{\prime}}{2}-\frac{1}{t t^{\prime}} \sin \frac{t}{2} \sin \frac{t^{\prime}}{2} \frac{1}{2\left(t+t^{\prime}\right)} \sin \frac{t+t^{\prime}}{2}-\frac{1}{t t^{\prime}} \sin \frac{t}{2} \sin \frac{t^{\prime}}{2} \\
\frac{1}{2\left(t+t^{\prime}\right)} \sin \frac{t+t^{\prime}}{2}-\frac{1}{t t^{\prime}} \sin \frac{t}{2} \sin \frac{t^{\prime}}{2} \frac{1}{2\left(t-t^{\prime}\right)} \sin \frac{t-t^{\prime}}{2}-\frac{1}{t t^{\prime}} \sin \frac{t}{2} \sin \frac{t^{\prime}}{2}
\end{array}\right) .
$$

Now it is straightforward to verify that the matrices $A^{\text {per }}$ and $A^{\text {cos }}$ satisfy

$$
\operatorname{Tr}\left[\tilde{A}^{\cos }\left(t_{1}, t_{2}\right) \tilde{A}^{\cos }\left(t_{2}, t_{3}\right) \cdots \tilde{A}^{\cos }\left(t_{l}, t_{1}\right)\right]=\operatorname{Tr}\left[A^{\mathrm{per}}\left(t_{1} / 2, t_{2} / 2\right) A^{\mathrm{per}}\left(t_{2} / 2, t_{3} / 2\right) \cdots A^{\mathrm{per}}\left(t_{l} / 2, t_{1} / 2\right)\right] .
$$

This establishes the validity of eqn (29).

Closed analytic expression have not been obtained for the above integrals. Actually the problem of computing the integrals in eqn (30) reduces to the solution of a homogeneous Fredholm equation of the second kind:

$$
\int_{0}^{\infty} \mathrm{d} t \sum_{j=1}^{2} \tilde{A}^{\cos }\left(t, t^{\prime}\right)_{i j} g_{j}\left(t^{\prime}\right)=\lambda g_{i}(t)
$$

where the $2 \times 2$ matrix $\tilde{A}^{\cos }\left(t, t^{\prime}\right)$ is a compact kernel with a discrete set of eigenvalues $\lambda_{k}$ converging to 0 , which encode all the information on the cumulants $\kappa_{l}$, in the same way as for the discrete case (eqn (23)).

The spectrum of the kernel $\tilde{A}^{\cos }\left(t, t^{\prime}\right)$ is most easily obtained by discretizing the variables $t$ and $t^{\prime}$ on an equally spaced grid with $t_{k}=\Delta\left(k-\frac{1}{2}\right), k=1, \ldots, N$, with an upper cut-off for the integrations. However, the singularities of the integrands makes the convergence rather slow $\left(\sim t_{n}^{2-\alpha}\right.$ for $t_{n} \rightarrow 0$ (with all other variables kept finite)). The divergence in the integrals at small $t$ is eliminated by a standard change of variables: an integral of a function diverging as $1 / t^{\gamma}$ for $t \rightarrow 0$ can be written as

$$
\int_{0}^{\infty} \mathrm{d} t f(t)=\frac{1}{1-\gamma} \int_{0}^{\infty} \mathrm{d} t t^{\gamma /(1-\gamma)} f\left[t^{1 /(1-\gamma)}\right] .
$$

The integrand on the right is constant for small $t$. Concretely, the change of variables in $\tilde{A}^{\text {cos }}$ leads to a matrix

$$
\tilde{\tilde{A}}^{\cos }\left(t, t^{\prime}\right)=\frac{1}{3-\alpha} t^{\frac{\alpha-2}{6-2 \alpha}} A^{\cos }\left(t^{1 /(3-\alpha)}, t^{\prime 1 /(3-\alpha)}\right) t^{\prime \frac{\alpha-2}{6-2 \alpha}},
$$

which can again be discretized. The characteristic function of the width distribution is computed from the spectrum of the $2 N \times 2 N$ matrix $\tilde{\tilde{A}}^{\cos }$ as discussed before. To show what is gained by rescaling the matrix $\tilde{A}$ in eqn (31), we have computed the second cumulant $\kappa_{2}$ on an equally spaced grid with both versions (see Fig. 6). The rescaled matrices converge exceptionally well with the discretization parameter $\Delta$. In Fig. 5, the spectrum of the rescaled kernel (for $\delta \rightarrow 0$ ) is compared to the spectra of $A^{\text {per }}$ and $A^{\cos }$ for finite $\delta$. Only in the special case $\alpha=2$ is the spectrum of the cosine series independent of $\delta$, because of the Markov-chain property of the random walk. For all $\alpha \neq 2$, the spectrum, thus the cumulants of the width distribution, depend of $\delta$, but satisfy

$$
\kappa_{l}^{\text {per }}(\delta \rightarrow 0)=\kappa_{l}^{\cos }(\delta \rightarrow 0) \neq \kappa_{l}^{\cos }(\delta=1),
$$

in a way illustrated in Fig. 5. As was already shown in Fig. 4 for $\alpha=2.5$, the convergence of the width distribution for finite $\delta$ towards the asymptotic width distribution is quite fast, even though the rate of convergence depends on the value of $\alpha$. 


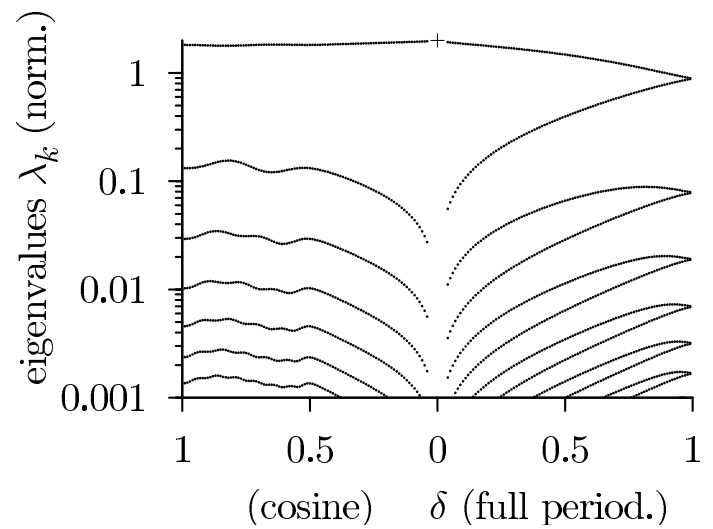

FIG. 7: Eigenvalue spectrum of $A^{\text {cos }}$ and $A^{\text {per }}$ for $\alpha=3.5(\zeta=1.25)$. A single eigenvalue remains non-zero in the limit $\delta \rightarrow 0$ (cross). The normalization condition $\lambda_{1}=\sum_{k} \lambda_{k}=2$ corresponds to the rescaling of the width distribution, with $\left\langle w_{2}\right\rangle=1$.

\section{Small-window limit $(\alpha \geq 3)$}

For $\alpha \geq 3$, the integral term in eqn (12) is sub-dominant, and the naive expansion of the matrices $A^{\text {per }}$ and $A^{\cos }$ in powers of $\delta$ becomes correct in the limit $\delta \rightarrow 0$. Using the expansions in eqn (18) and, furthermore,

$$
C_{m n}=O\left(\delta^{4}\right), \quad S_{m n}=\frac{1}{3} m n \pi^{2} \delta^{2}+O\left(\delta^{4}\right), \quad I_{n m}=O\left(\delta^{3}\right),
$$

we can construct the matrices $A^{\text {per }}$ and $A^{\text {cos }}$, and check that they have only a single non-zero eigenvalue. For illustration, we show in Fig. 7 the spectrum of these two matrices for $\alpha=7 / 2$ at finite $\delta$ (eigenvalues are normalized so that $\left.\sum_{k} \lambda_{k}=2\right)$.

From eqn (23) we then obtain

$$
\kappa_{l}^{\cos }(\delta \rightarrow 0)=\kappa_{l}^{\mathrm{per}}(\delta \rightarrow 0)= \begin{cases}(2 l-2) ! !+O\left(\delta^{\alpha-3}\right) & \text { for } \alpha>3 \\ (2 l-2) ! !+O(1 / \log \delta) & \text { for } \alpha=3\end{cases}
$$

The associated characteristic function reads:

$$
f(s)=(1-2 \mathrm{i} \lambda s)^{-\frac{1}{2}},
$$

and its inverse Fourier transform gives the universal distribution $\mathcal{P}(z)$ for $\alpha>3$,

$$
\mathcal{P}(z)=\frac{\exp [-z / 2]}{\sqrt{2 \pi z}} .
$$

By comparing the universal distribution for $\alpha>3$ with the ones with $\alpha<3$ (see Fig. 4), one notices that the additional eigenvalues (and branch points contributing to the characteristic function) allow the distribution $\mathcal{P}(z)$ to vanish at $z=0$, thus producing a local maximum in this function.

\section{CONCLUSIONS}

In conclusion, we have studied the geometric properties of functions with a particularly simple expression in Fourier space: independent Gaussian random variables. We have restricted ourselves to one-dimensional self-affine functions (characterized by a single length scale), but our analysis evidently carries over to functions with more than one length scale, and to higher dimensions. In real space, the geometrical properties are non-trivial, and the boundary conditions play an important role. This comes about because the real-space action, for all non-even $\alpha$, contains non-local operators and, for all $\alpha \neq 2$, is non-Markovian.

We have provided a simple and compact framework for studying the boundary effects for general non-Markovian Gaussian processes by relating the characteristic function of the width distribution to the spectrum of boundarydependent infinite matrices, which essentially encode the overlap of the basis functions of the Fourier series. The choice 
of Fourier basis (resulting in the full periodic, cosine and sine series) determines the different boundary conditions. We have carried out a complete analysis of the spectrum of these matrices for different values of $\alpha$ for the case of the function on the entire interval and also for the function restricted to a window. The associated width distributions could all be determined by solving for the eigenvalues of a matrix, and by performing a straightforward inverse Fourier transform.

We have shown that the non-Markovian action propagates the effects of boundary conditions over the entire interval. However, in the small-window limit, the width distribution becomes universal (independent of boundary conditions). For $\alpha<3$, we showed how to compute the cumulants of the width distribution in this limit, avoiding problems related to the non-uniform convergence of the Fourier series. For $\alpha>3$, the problem of finding the universal width distribution drastically simplifies, and we were able to write it down explicitly. Finally, we have obtained the logarithmic corrections in the case of odd integer $\alpha$, in particular for $\alpha=3$.

We hope that our work will be useful for the analysis of experimental data (which usually correspond to our window boundary conditions, often in the regime $\delta \rightarrow 0$, which we found to be universal). In many experiments, the roughness exponent cannot be extracted reliably by extrapolation, and the width distribution may provide crucial additional information.

Computer programs that compute the width distributions for any value of $\alpha$, both at finite $\delta$ and in the limit $\delta \rightarrow 0$, are available [27].

\section{Acknowledgments}

The authors thank the Kavli Institute for Theoretical Physics, Santa Barbara, for its hospitality. This research was supported in part by the National Science Foundation under the Grant No. PHY99-07949.

\section{APPENDIX A: EXPANSION FORMULA}

In this appendix we derive the expansion formula of eqn (12), first for non-integer, then for integer values of $\alpha$.

\section{Expansion formula for non-integer $\alpha$}

We consider the sum

$$
\sum_{n=1}^{\infty} \frac{f(n \delta)}{n^{\alpha}}
$$

where $f(z)$ is assumed to be a general analytic function. In order to obtain the expansion in powers of $\delta$ of the above sum, we expand $f(z)$ :

$$
\sum_{n=1}^{\infty} \frac{f(n \delta)}{n^{\alpha}}=\sum_{n=1}^{\infty} \sum_{m=0}^{\infty} \frac{f^{(m)}(0)}{m ! n^{\alpha-m}} \delta^{m}
$$

For $\alpha-m<1$, the sum over $n$ converges, and can be directly evaluated using the Riemann Zeta function $\zeta(\alpha-m)=$ $\sum_{n} 1 / n^{\alpha-m}:$

$$
\sum_{n=1}^{\infty} \frac{f(n \delta)}{n^{\alpha}}=\sum_{m=0}^{[\alpha]-1} \frac{f^{(m)}(0) \zeta(m-\alpha)}{m !} \delta^{m}+\sum_{n=1}^{\infty} \frac{\tilde{f}(n \delta)}{n^{\alpha}},
$$

where $[\alpha]$ is the integer part of $\alpha$, and $\tilde{f}(z)=\sum_{[\alpha]}^{\infty} f^{(m)}(0) z^{m} / m !$. The second term on the right hand side of eqn (A1) can be expressed as a contour integral in the complex plane:

$$
\sum_{n=1}^{\infty} \frac{\tilde{f}(n \delta)}{n^{\alpha}}=\frac{\delta^{\alpha-1}}{2 \mathrm{i}} \int_{C} \mathrm{~d} z \frac{\tilde{f}(z)}{z^{\alpha}} \cot \left(\frac{\pi}{\delta} z\right)
$$

where the contour $C$ encircles the poles $z_{n}=n \delta, n=1,2 \ldots$ of the function $G(z)=\left[\tilde{f}(z) / z^{\alpha}\right] \cot (\pi z / \delta)$. The contour $C$ is transformed into the contour $C_{\epsilon}$, as shown in Fig. 8, avoiding the branch point at the origin. By considering the 

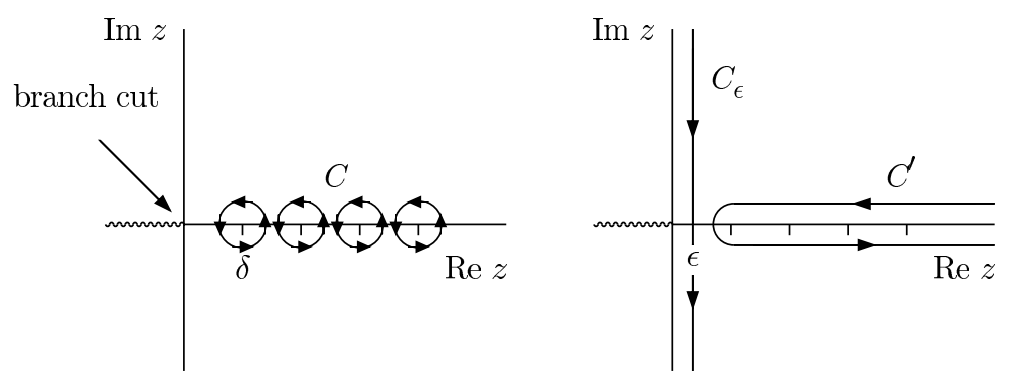

FIG. 8: Contours for the integral in eqn (A2). The indicated deformation of the contour $\left(C \rightarrow C^{\prime} \rightarrow C_{\epsilon}\right)$ transforms eqn $($ A2) into eqn (A3).

integration over the contour $C_{\epsilon}$, and by performing the change of variables $z \rightarrow-\mathrm{i}(z / \delta-\epsilon)$, we have:

$$
\sum_{n=1}^{\infty} \frac{\tilde{f}(n \delta)}{n^{\alpha}}=-\frac{\mathrm{i}}{2} \int_{-\infty}^{\infty} \mathrm{d} z \frac{\tilde{f}[\delta(\mathrm{i} z+\epsilon)]}{(\mathrm{i} z+\epsilon)^{\alpha}} \operatorname{coth}[\pi(z-\mathrm{i} \epsilon)] .
$$

To extract the $\delta$-series of the above integral, it is tempting to integrate term by term the Taylor series of the function $\tilde{f}(z)$. We first split the above integral into the following terms:

$$
\begin{aligned}
\sum_{n=1}^{\infty} \frac{\tilde{f}(n \delta)}{n^{\alpha}}=-\frac{\mathrm{i}}{2} \int_{0}^{\infty} \mathrm{d} z \tilde{f}[\delta(\mathrm{i} z+\epsilon)](\mathrm{i} z+\epsilon)^{-\alpha}[\operatorname{coth}[\pi(z-\mathrm{i} \epsilon)]-1]-\frac{\mathrm{i}}{2} \int_{0}^{\infty} \mathrm{d} z \tilde{f}[\delta(\mathrm{i} z+\epsilon)](\mathrm{i} z+\epsilon)^{-\alpha} \\
\quad-\frac{\mathrm{i}}{2} \int_{-\infty}^{0} \mathrm{~d} z \tilde{f}[\delta(\mathrm{i} z+\epsilon)](\mathrm{i} z+\epsilon)^{-\alpha}[\operatorname{coth}[\pi(z-\mathrm{i} \epsilon)]+1]+\frac{\mathrm{i}}{2} \int_{-\infty}^{0} \mathrm{~d} z \tilde{f}[\delta(\mathrm{i} z+\epsilon)](\mathrm{i} z+\epsilon)^{-\alpha} .
\end{aligned}
$$

This separation is valid if all the above integrals are well-defined, a condition respected by the functions $f(z)$ considered in this paper (for $\delta \leq 1$ ). Using the following representation of the Riemann Zeta function, valid for $\operatorname{Re}(\beta)<1$ :

$$
\zeta(\beta)=\lim _{\epsilon \rightarrow 0}\left(-\frac{\mathrm{i}}{2}\left[\int_{0}^{\infty} \mathrm{d} z(\mathrm{i} z+\epsilon)^{-\beta}(\operatorname{coth}[\pi(z-\mathrm{i} \epsilon)]-1)+\int_{-\infty}^{0} \mathrm{~d} z(\mathrm{i} z+\epsilon)^{-\beta}(\operatorname{coth}[\pi(z-\mathrm{i} \epsilon)]+1)\right]\right),
$$

we obtain

$$
\sum_{n=1}^{\infty} \frac{\tilde{f}(n \delta)}{n^{\alpha}}=\sum_{m=[\alpha]}^{\infty} \frac{f^{(m)}(0)}{m !} \delta^{m} \zeta(\alpha-m)+\frac{-\mathrm{i}}{2} \int_{0}^{\infty} \mathrm{d} z \tilde{f}(z)(\mathrm{i} z+\epsilon)^{-\alpha}+\frac{\mathrm{i}}{2} \int_{-\infty}^{0} \mathrm{~d} z \tilde{f}(z)(\mathrm{i} z+\epsilon)^{-\alpha} .
$$

Finally, by considering the contours shown in Fig. 9, we verify that

$$
\lim _{\epsilon \rightarrow 0}-\frac{\mathrm{i}}{2} \int_{0}^{\infty} \mathrm{d} z \tilde{f}(\delta(\mathrm{i} z+\epsilon))(\mathrm{i} z+\epsilon)^{-\alpha}+\frac{i}{2} \int_{-\infty}^{0} \mathrm{~d} z \tilde{f}(\delta(\mathrm{i} z+\epsilon))(\mathrm{i} z+\epsilon)^{-\alpha}=\delta^{\alpha-1} \int_{0}^{\infty} \mathrm{d} t \frac{\tilde{f}(t)}{t^{\alpha}} .
$$

Collecting these results proves eqn (12).

\section{Expansion formula for integer $\alpha$}

The series in eqn (12) is not defined for integer $\alpha=m+1$, because of the simple pole of the Riemann Zeta function $\zeta(z)$ at $z=1$. However, this divergence is compensated by an ultraviolet divergence in the integral. We analyze this situation by considering $\alpha=\lfloor\alpha\rfloor+\rho$, and by taking the limit $\rho \rightarrow 0$. We write:

$$
\delta^{\lfloor\alpha\rfloor-1+\rho} \int_{0}^{\infty} \mathrm{d} t\left(\frac{f(t)}{t^{\alpha}}-\sum_{m=0}^{\lfloor\alpha\rfloor-1} \frac{f^{m}(0) t^{m-\lfloor\alpha\rfloor+\rho}}{m !}\right)+\delta^{\lfloor\alpha\rfloor-1} \frac{f^{(\alpha-1)}(0)}{(\alpha-1) !} \zeta(1+\rho) .
$$

In order to isolate the ultraviolet divergence, we split the integral into two infrared-divergent terms, and eqn (A5) becomes

$$
\delta^{\lfloor\alpha\rfloor+\rho-1} \int_{\epsilon}^{\infty} \mathrm{d} t\left(\frac{f(t)}{t^{\alpha}}-\sum_{m=0}^{\lfloor\alpha\rfloor-2} \frac{f^{(m)}(0) t^{m-\alpha}}{m !}\right)-\delta^{\lfloor\alpha\rfloor+\rho-1} \int_{\epsilon}^{\infty} \mathrm{d} t \frac{f^{(\alpha-1)}(0)}{(\alpha-1) ! t^{1+\rho}}+\delta^{\lfloor\alpha\rfloor-1} \frac{f^{(\alpha-1)}(0)}{(\alpha-1) !} \zeta(1+\rho),
$$




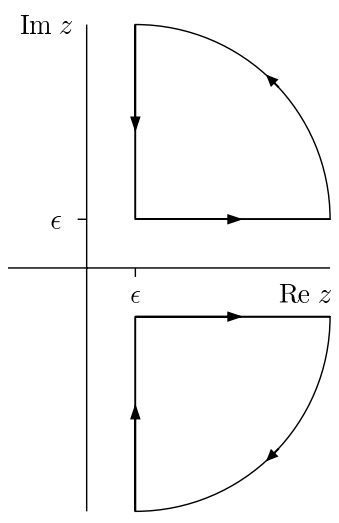

FIG. 9: Contours of the integrals in eqn (A4).

where $\epsilon$ is an infrared cut-off. Using the expansion $\zeta(1+\rho)=1 / \rho+\gamma+O(\rho)$, where $\gamma$ is the Euler constant, we can take the limit $\rho \rightarrow 0$ :

$$
\lim _{\rho \rightarrow 0}\left[\zeta(1+\rho)-\delta^{\rho} \int_{\epsilon}^{\infty} \mathrm{d} t \frac{1}{t^{1+\rho}}\right]=[\gamma-\log (\delta)-\log (\epsilon)] .
$$

From the above we get the following expansion formula for integer values of $\alpha$ :

$$
\sum_{n=1}^{\infty} \frac{f(n \delta)}{n^{\alpha}}=\delta^{\alpha-1}\left(\text { const }-\frac{f^{(\alpha-1)}(0)}{(\alpha-1) !} \log (\delta)\right)+\sum_{m \neq \alpha-1} \delta^{m} f^{(m)}(0) \frac{\zeta(\alpha-m)}{m !},
$$

where the constant is expressed in term of the following limit:

$$
\text { const }=\lim _{\epsilon \rightarrow 0}\left(\int_{\epsilon}^{\infty} \mathrm{d} t\left(\frac{f(t)}{t^{\alpha}}-\sum_{m=0}^{\alpha-2} \frac{f^{(m)}(0) t^{m-\alpha}}{m !}\right)+\frac{f^{(\alpha-1)}(0)}{(\alpha-1) !}(\log (\epsilon)+\gamma)\right) .
$$

\section{APPENDIX B: GENERAL CUMULANTS}

For completeness, we derive in this appendix the trace formula for the cumulants $\kappa_{l}$ of the rescaled width distribution $\Psi(s)$ for all $l$ from their generating function

$$
\Psi(s)=\log \left\langle\exp \left(s w_{2}\right)\right\rangle=\sum_{l=1}^{\infty} \frac{\kappa_{l}}{l !} s^{l} .
$$

We first consider the full periodic series. To compute the average $\left\langle\exp \left(s w_{2}\right)\right\rangle$ we have to integrate over the Gaussians $\left\{a_{n}, b_{n}\right\}$. To make all the Gaussian integrals well-defined, we introduce a cut-off $N$ in the momentum space, such that $n=1, \ldots, N$. The average $\left\langle\exp \left(s w_{2}\right)\right\rangle$ takes the form:

$$
\left\langle\exp \left(s w_{2}^{\mathrm{per}}\right)\right\rangle=\frac{\left(\prod_{n^{\prime}} \int_{-\infty}^{\infty} \mathrm{d} x_{n^{\prime}}\right) \exp \left[-\frac{1}{2} \sum_{n^{\prime} m^{\prime}} M_{n^{\prime} m^{\prime}}(s) x_{n^{\prime}} x_{m^{\prime}}\right]}{\left(\prod_{n^{\prime}} \int_{-\infty}^{\infty} \mathrm{d} x_{n^{\prime}}\right) \exp \left[-\frac{1}{2} \sum_{n^{\prime} m^{\prime}} M_{n^{\prime} m^{\prime}}(0) x_{n^{\prime}} x_{m^{\prime}}\right]}=\left[\frac{\operatorname{Det} M(s)}{\operatorname{Det} M(0)}\right]^{-\frac{1}{2}},
$$

where the indices $n^{\prime}$ and $m^{\prime}$ run from 0 to $2 N$. In the above expression, we set $x_{n^{\prime}}=a_{n}^{\prime}$, for $n^{\prime}=0, \ldots, N$ and $x_{n^{\prime}}=b_{n^{\prime}-N}$ for $n^{\prime}=N+1, \ldots, 2 N$. The matrix $M(s)$ is a block of four $N \times N$ matrices defined as

$$
M(s)=\left(\begin{array}{cc}
\sigma_{n}^{-2} \delta_{n m} & 0 \\
0 & \sigma_{n}^{-2} \delta_{n m}
\end{array}\right)+s\left(\begin{array}{cc}
2 C_{n m} & I_{n m} \\
I_{m n} & 2 S_{n m}
\end{array}\right)
$$

(for the coefficients $C_{n m}, I_{n m}$, and $S_{n m}$, see eqn (9)). We arrive at the following expression for the $\Psi(s)$ :

$$
\Psi(s)=-\frac{1}{2} \log \operatorname{Det} M(s)+\frac{1}{2} \log \operatorname{Det} M(0)=-\frac{1}{2} \operatorname{Tr}[\log M(s)]+\frac{1}{2} \operatorname{Tr}[\log M(0)] .
$$


Expanding the logarithm $\log M(s)$ one obtains

$$
\Psi\left(s /\left\langle w_{2}\right\rangle\right)=\sum_{l=1}^{\infty} \frac{\left(s /\left\langle w_{2}\right\rangle\right)^{l}}{l} \operatorname{Tr}\left[\left(A^{\mathrm{per}}\right)^{l}\right]
$$

where $A^{\text {per }}$ is given in eqn (21). Hence the $l$ th cumulant of the roughness is given by

$$
\kappa_{l}^{\mathrm{per}}=\frac{(l-1) !}{2\left\langle w_{2}\right\rangle^{l}} \operatorname{Tr}\left[\left(A^{\mathrm{per}}\right)^{l}\right]
$$

For the cosine series, one has to integrate over the Gaussian variables $\left\{c_{n}\right\}$ and $\left\{s_{n}\right\}$. One arrives at an analogous expression for the cumulants.

[1] Z. Toroczkai and E. D. Williams, Phys. Today 52, 24 (1999)

[2] M. Kardar, Phys. Rep. 301, 85 (1998).

[3] U. Frisch, Turbulence: The Legacy of A. N. Kolmogorov (Cambridge University Press, Cambridge, UK, 1995).

[4] Proceedings of the 15th International Conference on Noise in Physical Systems and 1/f Fluctuations, edited by C. Surya, IEEE Proceedings, 1999.

[5] A. Rosso, W. Krauth, P. Le Doussal, J. Vannimenus, and K. J. Wiese, Phys. Rev. E 68, 036128 (2003).

[6] P. Le Doussal and K. J. Wiese, Phys. Rev. E 68, 046118 (2003).

[7] K. B. Oldham and J. Spanier, The Fractional Calculus: Integrations and Differentiations of Arbitrary Order (Academic Press, New York, 1974).

[8] S. N. Majumdar, Current Science 89, 2076 (2005).

[9] S. V. Khare and T. L. Einstein, Phys. Rev. B 57, 4782 (1998)

[10] S. N. Majumdar and A. J. Bray, Phys. Rev. Lett. 86, 3700 (2001).

[11] S. N. Majumdar and A. Comtet, Phys. Rev. Lett. 92, 225501 (2004).

[12] S. L. A. de Queiroz, Phys. Rev. E 71, 016134 (2005).

[13] S. Moulinet, A. Rosso, W. Krauth, and E. Rolley, Phys. Rev. E 69, 035103(R) (2004).

[14] R. P. Feynman, Statistical Mechanics (Addison-Wesley, New York, 1972).

[15] W. Krauth, Statistical Mechanics: Algorithms and Computations (Oxford University Press, Oxford, 2006) (See www.phys.ens.fr/doc/SMAC).

[16] A. Rosso, R. Santachiara, and W. Krauth, J. Stat. Mech. L08001 (2005).

[17] G. Foltin, K. Oerding, Z. Rácz, R. L. Workman, and R. K. P. Zia, Phys. Rev. E 50, R639 (1994).

[18] M. Plischke, Z. Rácz, and R. K. P. Zia, Phys. Rev. E 50, 3589 (1994).

[19] T. Antal, M. Droz, G. Györgyi, and Z. Rácz, Phys. Rev. E 65, 046140 (2001).

[20] S. T. Bramwell and et al., Phys. Rev. Lett. 84, 3744 (2000).

[21] G. Parisi, Z. Rácz, E. Marinari, and A. Pagnani, Phys. Rev. E 69, 026136 (2002).

[22] D. Vandembroucq and S. Roux, Phys. Rev. E 70, 026103 (2004).

[23] S. Zapperi, P. K. V. V. Nukala, and S. Šimunović, Phys. Rev. E 71, 026106 (2005).

[24] H. Leschhorn and L. Tang, Phys. Rev. Lett. 70, 2973 (1993).

[25] H. J. Jensen, J. Phys. A 28, 1861 (1995).

[26] A. Rosso and W. Krauth, Phys. Rev. Lett. 87, 187002 (2001).

[27] See www.lps.ens.fr/ krauth 